\title{
A case report of dyslipidemia management in new-onset type 2 diabetic patient
}

\author{
Danish Mahmood* \\ Department of Pharmacology and Toxicology, Unaizah College of Pharmacy, Qassim University, Kingdom of Saudi Arabia
}

\begin{abstract}
New-onset diabetes is a serious condition and is characterised by insulin resistance and dyslipidemia affecting adults and more lately children. Diabetic dyslipidemia is characterised by high plasma triglycerides and low-density lipoprotein cholesterol (LDL-C) particles, and low high-density lipoprotein cholesterol (HDL-C). Individuals with dyslipidemia have a high 10-year risk of atherosclerotic cardiovascular disease (ASCVD) and can be managed by adopting therapeutic lifestyle changes (TLC) and medication adherence. The new American College of Cardiology/American Heart Association (ACC/AHA) guideline emphasizes on matching the intensity of statin treatment to the level of ASCVD risk and replaces the old paradigm of LDL-C target based treatment, and the new guidelines of National Lipid Association (NLA) have recommended a multifaceted patient centered approach for the management of dyslipidemia. Both ACC/AHA and NLA have recommended statins as the main stay of drug therapy in patients with high cholesterol. Unlike ACC/AHA guidelines who do not recommend the use of nonstatin drugs except in the statin-intolerant patients, the NLA advocates nonstatins use in high-risk patients, and in patients with inherited cholesterol disorders, after statin therapy. Medication non adherence is a frequent problem in developing countries because of the low income and high cost of drugs which are sometimes required for a long duration of time. TLC has been reported to aid drug therapy to provide best medication benefit but lack of knowledge and illiteracy is another limitation to achieve maximum benefits from drug therapy in India.
\end{abstract}

In conclusion, both TLC and medication adherence are important for dyslipidemia management with lipid lowering drugs in new-onset type 2 diabetic patient, and healthcare providers should provide knowledge on the benefits of TLC and medication adherence in countries with low income/socio-economic status.

\section{Introduction}

New-onset diabetes is a serious condition that adversely affects the body's ability to regulate glucose. The new-onset diabetes is characterized by a form of insulin resistance that historically affected individuals in adulthood. In recent years, however, diagnoses of this disease have become prevalent in children. It is often observed that people who are obese and insulin resistant often have a dyslipidemia. Dyslipidemia is commonly characterized by elevated blood triglyceride (TG) concentrations and low high-density lipoprotein cholesterol (HDL-C) [1]. These characteristic are also considered important cardio-metabolic risk factors, increase the risk of macrovascular and microvascular complications in type 2 diabetes for the development of cardiovascular diseases [2]. Dyslipidemia consisting of high TGs and low HDL-C is a widely recognized lipid pattern that is frequently associated with the development of coronary heart disease. Study has suggested that dyslipidemia in diabetic patients should be treated for primary prevention with the same intensity recommended for secondary prevention in heart patients, before the development of cardiovascular disease [3]. Strict adherence to treatment is essential to avail maximum benefits of therapy. Further, Therapeutic Lifestyle changes (TLC) have been reported to reduce LDL-C, TG, blood pressure, glucose and body weight and increase HDL-C level and have greatly complemented the drug therapy [4]. Therefore, strict adherence to the prescribed therapy and TLC are key to obtain optimum therapeutic benefits. In poor countries like India and others, socio-economic states are major determinant of medication continuation and play an important role in deciding adherence rate. There has been a significant association between patients with low-income and non-adherence to drugs, and high cost of newer oral hypoglycaemic agents have hindered optimal adherence to the treatment [5].
We present here a case report of effective management of dyslipidemia in a new-onset type 2 diabetic with TLC and strict adherence to pharmacological treatments.

\section{Case report}

A 60-year old man with new-onset, type 2 diabetes mellitus visited a tertiary care hospital in India. His weight and height was $80 \mathrm{~kg}$ and 5'9", respectively. The patient had a body mass index of $26 \mathrm{~kg} / \mathrm{m}^{2}$ and blood pressure measured as $140 / 90 \mathrm{~mm} \mathrm{Hg}$. He had a sedentary lifestyle, mild polyphagia, and presented with nonspecific complaints of fatigue and lack of general sense of well-being. The patient had strong positive family history of cardiovascular diseases (CVD), his father had myocardial infarction (MI) which proved to be fatal, mother and elder sister had type 2 diabetes and younger brother had an ischemic stroke 5 years ago. Laboratory findings showed total cholesterol as $227 \mathrm{mg} /$ dL, HDL-C levels as $40 \mathrm{mg} / \mathrm{dL}$, LDL- as $125 \mathrm{mg} / \mathrm{dL}$, TG as $140 \mathrm{mg} /$ $\mathrm{dL}$, fasting blood glucose as $104 \mathrm{mg} / \mathrm{dL}$, and glycated haemoglobin (HbA1C) as $8 \%$. Patient was taking metformin plus atorvastatin (500 $\mathrm{mg}+10 \mathrm{mg}$, once daily) for past 3 months but had poor adherence to treatment. His 10-year risk of atherosclerotic cardiovascular disease (ASCVD) risk was calculated as $23.5 \%$ using pooled cohort risk assessment equations.

Correspondence to: Danish Mahmood, Department of Pharmacology and Toxicology, Unaizah College of Pharmacy, Qassim University, Kingdom of Saudi Arabia, Tel: +966594016751; E-mail: ma.alam@qu.edu.sa

Key words: dyslipidemia, LDL-C, TLC, diabetes, ACC/AHA, ASCVD

Received: February 14, 2015; Accepted: March 17, 2015; Published: March 21, 2015 
After strict adherence to the prescribed treatment (metformin and atorvastatin) and lifestyle modifications, his $\mathrm{HbA1C}$ level improved to $7 \%$ and LDL-C dropped to $73 \mathrm{mg} / \mathrm{dL}$ at 3-month follow-up.

However, there was no significant improvement in HDL-C and TG levels after initiation of atorvastatin. During treatment, the clinician increased the atorvastatin dose to $80 \mathrm{mg}$ /day which further reduced LDL-C level. The high atorvastatin dose was well-tolerated by the patient with no elevations of alanine/aspartate aminotransferase. At 6-month follow-up, the LDL-C level again rose to $85 \mathrm{mg} / \mathrm{dL}$, which was found to be due to medication noncompliance.

\section{Discussion}

The present case report highlights how life style modification and strict adherence to treatment can effectively manage diabetic dyslipidemia in type 2 diabetes.

Lifestyle changes, including increased physical activity and dietary modifications remains the cornerstone of management of atherogenic dyslipidemia in type 2 diabetes [6-8]. In our report, when the lifestyle of the patient was modified and he strictly adhered to the treatment as suggested by the physician, his HbAlc and LDL-C levels significantly improved.

Our findings are in concordance with earlier reported literature where intensive lifestyle interventions yielded better long-term impact on cardiovascular morbidity and mortality in overweight patients with type 2 diabetes than standard diabetes support and education programs [9]. Similarly, stepwise introduction of lifestyle modification with pharmacologic interventions helped to reduce the risk of deaths (53\%) due to CVD, nonfatal MI, percutaneous coronary interventions, nonfatal stroke, and coronary artery bypass grafting in patients with type 2 diabetes and microalbuminuria [10]. Metformin is most prescribed oral antidiabetic agent [11]. Statins, the lipid-lowering therapy, is the recommended initial pharmacological treatment for lowering LDL-C levels in "very high risk" and "high-risk" categories of type 2 diabetes. Atorvastatin is one of the most worldwide prescribed statins [12].

Looking at the calculated 10 year risk of ASCVD of the patient, he was at an elevated 10 -year risk ( $\geq 7.5 \%$ ) for ASCVD. As per the Pooled Cohort Risk Assessment Equation, a high-intensity statin should be considered for this patient who has a 10 -year ASCVD risk $\geq 7.5 \%$ and fall in the age bracket of 40-75 years and also have LDL 70-189 mg/dL. The 2013 American College of Cardiology (ACC)/American Heart Association (AHA) have recommended either a high-intensity or moderate-intensity statin regimen in patients who have an elevated ASCVD risk $(\geq 7.5 \%)$ for primary prevention of cardiovascular disease [13]. The new ACC/AHA guideline emphasizes matching the intensity of statin treatment to the level of ASCVD risk and replaces the old paradigm of pursuing LDL-C targets. While, the new guidelines of National Lipid Association (NLA) have recommended a multifaceted approach to incorporate each patient risk and risk factors, rather than specific medication categories for the management of dyslipidemia. The NLA recommendations differ from the ACC/AHA guidelines in that it continued the use of lipid-goals and non-HDL treatment goals, as it considers non-HDL to be a better predictor of ASCVD morbidity and mortality than LDL. Also, unlike ACC/AHA, NLA do not advocate the use of a particular risk calculator. An elevated level of atherogenic cholesterol is regarded as the root cause of atherosclerosis, the key underlying process contributing to most clinical ASCVD. Hence, reducing elevated levels of atherogenic cholesterol particles has been recommended to lower ASCVD and can be achieved by atherogenic cholesterol lowering through multiple modalities, including lifestyle and drug therapies. The intensity of risk-reduction therapy should be individualised based on the patient's absolute risk for an ASCVD event and, both intermediate-term and long-term/lifetime risk should be considered when assessing the potential benefits and hazards of riskreduction therapies [14]. Both NLA and ACC/AHA recommend statin treatment as the mainstay of drug therapy to reduce ASCVD risk in patients who have been considered for treatment with lipid-lowering drug therapy. Further, NLA recommends management of nonlipid ASCVD risk factors particularly high blood pressure, cigarette smoking, and diabetes mellitus.

The ACC/AHA have recommended $80 \mathrm{mg}$ (40 less preferred) dose of high intensity atorvastatin. Hence, atorvastatin dose was increased from $10 \mathrm{mg}$ to $80 \mathrm{mg}$. He was advised to adopt TLC as the 2013 ACC. AHA guideline have laid extra emphasis on lifestyle modification (i.e., adhering to a heart healthy diet, regular exercise habits, avoidance of tobacco products, and maintenance of a healthy weight). TLC constitutes an important element in the health promotion and ASCVD risk reduction, both prior to and in concert with the use of cholesterol lowering drug therapies. TLC is a comprehensive lifestyle approach thatincludes specific dietary recommendations (TLCdiet), weight management, and increased physicalactivity. The TLC emphasizes reducing dietary cholesterol ( $<200 \mathrm{mg} /$ day), saturated fats ( $<7 \%$ of total calories), and trans fats (lower intake). Total fat comprises $25-35 \%$ of total calories, with up to $20 \%$ coming from monounsaturated fats and $10 \%$ from polyunsaturated fats. The optional nutrient considerations for augmented LDL-C reduction and reducing coronaryheart disease risk include stanols/sterols ( $2 \mathrm{~g} /$ day) and viscous (soluble) fibers (5-10 $\mathrm{g} /$ day), and including fish (especially oily fish such as salmon, tuna, and mackerel) as part ofone's overall eating plan [4].

After the use of high intensity atorvastatin dose $(80 \mathrm{mg} /$ day $)$, his significantly LDL-C reduced with no adverse events indicating that the dose was safe. The diabetes atorvastatin lipid intervention (DALI) study have reported that either 10 or $80 \mathrm{mg}$ of atorvastatin is equally effective in the treatment of diabetic dyslipidemia [15]. In 2008, Balasubramanian et al. in a study on Indian patients assessed the efficacy, safety and tolerability of a fixed dose combination of atorvastatin $10 \mathrm{mg}+$ metformin SR $500 \mathrm{mg}$ in adult Indian patients with diabetic dyslipidaemia. Further, increased dose of atorvastatin was safe in the present case [16]. This was consistent with previous study reporting that atorvastatin initiated at doses of 10,20,40, and $80 \mathrm{mg}$ was effective and safe for the treatment of patients with dyslipidemia. It was further stated that the dose of atorvastatin should depend on the percentage reduction needed to achieve an LDL-C goal, patients with or at risk of coronary heart disease may benefit from starting therapy at a higher dose of atorvastatin [17].

Our results were parallel with the earlier reported literature where high-dose statins led to significant reduction in the mean LDL-C levels and in the occurrence of major $\mathrm{CV}$ events (CHD, stroke, revascularizations) in comparison to low-dose statins [18]. In 2006, Shepherd et al. [19] reported high-dose statin monotherapy as safe with no treatment-related adverse events and no elevated levels of liver enzymes.

In developing and poor economic countries including India, there continues to be low medical education and awareness on effective usage of medications prescribed by doctors. The illiterate and poor patients tend to abruptly quit medication or do not stick to the prescribed dosage regimen of the medication leading to suboptimal 
use and clinical outcome. Hence, these patients remain undertreated for diseases that can be effectively treated. The interpretation of a recent study in patients with rheumatoid arthritis suggested that patients who were not taking their biological drug on the day agreed with their health-care professional had poorer clinical outcomes than those who did take their drug, emphasising the need for strict adherence to biological therapy in patients with this condition [20].

Medication adherence commonly refers to whether patients take their medications as prescribed (e.g., twice daily), as well as whether they continue to take a prescribed medication, and it is defined as patients who is taking at least $80 \%$ of the amount of medication given to him, and is based on days supply of medication divided by the number of days patient should be consuming the medication. This is termed as the medication possession ratio (MPR). Patient with $>80 \%$ MPR is regarded as poor adherence. Recent study have suggested even MPR $\geq 90 \%$ to be a better threshold for considering patient as adherent [21]. Nonadherence to the prescribe therapy is a growing concern to clinicians, healthcare systems, and other stakeholders (eg, payers) because of mounting evidence that it is prevalent and associated with adverse outcomes and higher costs of care [22]. It potentially leads to increase in hospital visit, duration of hospital stay, suboptimal therapeutic benefit and costs of treatment, and it is likely to grow worldwide as patients often take more medications to treat chronic conditions and is common for patients with CVDs. In this case, we have observed that due to medication nonadherence, patient's LDL-C levels had shoot up from 75 to $85 \mathrm{mg} / \mathrm{dL}$ and the beneficial effects of treatment were abolished. Similar results in terms of higher risk of death, nonfatal MI and loss of beneficial effects of statins with abrupt discontinuation were reported in patients with acute coronary syndrome [22].

\section{Conclusion}

There continues to be a considerable lack of knowledge among people in developing countries on dyslipidemia which can be effectively managed in type 2 diabetes patients by life-habit modifications such as adoption of TLC in combination with strict adherence to pharmacological interventions. Therefore, there is a need to impart awareness on the dosage, potential side effects, benefits of continuing uninterrupted medication, by clinicians and pharmacists. This would surely translate into an effective and optimised management of dyslipidemia and would potentially decrease the occurrence of dyslipidemia diabetic patients. Hence, health providers in should underscore the benefits of continuing and adhering to the prescribed statin therapy including TLC.

\section{References}

1. Mooradian AD (2009) Dyslipidemia in type 2 diabetes mellitus. Nat Clin Pract Endocrinol Metab 5: 150-159. [Crossref]

2. Farmer JA (2008) Diabetic dyslipidemia and atherosclerosis: evidence from clinica trials. CurrDiab Rep 8: 71-77. [Crossref]

3. Grover SA, Coupal L, Zowall H, Weiss TW, Alexander CM (2003) Evaluating the benefits of treating dyslipidemia: the importance of diabetes as a risk factor. $\mathrm{Am} \mathrm{J} \mathrm{Med}$ 115: 122-128. [Crossref]

4. http://www.cdc.gov/nutrition/downloads/r2p_life_change.pdf; accessed on 10th April 2015.
5. Sharma T, Kalra J, Dhasmana DC, Basera H (2014) Poor adherence to treatment: A major challenge in diabetes. JIACM 15: 26-29.

6. Kataokam Y, Uno K, Nicholls SJ (2011) Management of Dyslipidemia in Patients with Diabetes. US Endocrinol 7: 40-45.

7. American Diabetes Association, Bantle JP, Wylie-Rosett J, Albright AL, Apovian CM, et al. (2008) Nutrition recommendations and interventions for diabetes: a position statement of the American Diabetes Association. Diabetes Care 31 Suppl 1: S61-S78. [Crossref]

8. (2002) National Cholesterol Education Program (NCEP) Expert Panel on Detection, Evaluation, and Treatment of High Blood Cholesterol in Adults (Adult Treatment Pane III). Third Report of the National Cholesterol Education Program (NCEP) Expert Panel on Detection, Evaluation, and Treatment of High Blood Cholesterol in Adults (Adult Treatment Panel III): final report. Circulation 106: 3143-3421.

9. Look AHEAD Research Group, Pi-Sunyer X, Blackburn G, Brancati FL, Bray GA, et al. (2007) Reduction in weight and cardiovascular disease risk factors in individuals with type 2 diabetes: one-year results of the look AHEAD trial. Diabetes Care 30: 1374-1383. [Crossref]

10. Gaede P, Vedel P, Larsen N, Jensen GV, Parving HH, et al. (2003) Multifactorial intervention and cardiovascular disease in patients with type 2 diabetes. $N$ Engl J Med 348: 383-393. [Crossref]

11. Rojas LB, Gomes MB (2013) Metformin: an old but still the best treatment for type 2 diabetes. Diabetol Metab Syndr 5: 6. [Crossref]

12. Save V, Patil N, Moulik N, Rajadhyaksha G (2006) Effect of atorvastatin on type 2 diabetic dyslipidemia. J Cardiovasc Pharmacol Ther 11: 262-270. [Crossref]

13. Ray KK, Kastelein JJ, Boekholdt SM, Nicholls SJ, Khaw KT, et al. (2014) The ACC/ AHA 2013 guideline on the treatment of blood cholesterol to reduce atherosclerotic cardiovascular disease risk in adults: the good the bad and the uncertain: a comparison with ESC/EAS guidelines for the management of dyslipidaemias 2011. Eur Heart $J$ 35:960-8. [Crossref]

14. Jacobson TA, Ito MK, Maki KC, Orringer CE, Bays HE, et al. (2014) National Lipid Association recommendations for patient-centered management of dyslipidemia: part 1-executive summary. J Clin Lipidol 8: 473-488. [Crossref]

15. The Diabetes Atorvastatin Lipid Intervention (DALI) Study Group (2001) The effect of aggressive versus standard lipid lowering by atorvastatin on diabetic Dyslipidemia: the DALI study: a double-blind, randomized, placebo-controlled trial in patients with type 2 diabetes and diabetic dyslipidemia. Diabetes Care 24: 1335-1341. [Crossref]

16. Balasubramanian R, Varadharajan S, Kathale A, Nagraj LM, Periyandavar I, et al. (2008) Assessment of the efficacy and tolerability of a fixed dose combination of atorvastatin $10 \mathrm{mg}+$ metformin SR $500 \mathrm{mg}$ in diabetic dyslipidaemia in adult Indian patients. J Indian Med Assoc 106: 464-467. [Crossref]

17. Jones PH, McKenney JM, Karalis DG, Downey J (2005) Comparison of the efficacy and safety of atorvastatin initiated at different starting doses in patients with dyslipidemia Am Heart J 149: e1. [Crossref]

18. LaRosa JC, Grundy SM, Waters DD, Shear C, Barter P, et al. (2005) Intensive lipid lowering with atorvastatin in patients with stable coronary disease. $N$ Engl J Med 352 : 1425-1435. [Crossref]

19. Shepherd J, Barter P, Carmena R, Deedwania P, Fruchart JC, et al. (2006) Treating to New Targets Investigators. Effect of lowering LDL-C cholesterol substantially below currently recommended levels in patients with coronary heart disease and diabetes: the Treating to New Targets (TNT) study. Diab Care 29: 1220-226.

20. Ho PM, Bryson CL, Rumsfeld JS (2009) Medication adherence: its importance in cardiovascular outcomes. Circulation 119: 3028-3035. [Crossref]

21. Watanabe JH, Bounthavong M, Chen T (2013) Revisiting the medication possession ratio threshold for adherence in lipid management. Curr Med Res Opin 29: 175-180. [Crossref]

22. Spencer FA, Fonarow GC, Frederick PD, Wright RS, Every N, et al. (2004) Early withdrawal of statin therapy in patients with non-ST-segment elevation myocardial infarction: national registry of myocardial infarction. Arch Intern Med 164: 2162-2168. [Crossref]

Copyright: (C2015 Mahmood D. This is an open-access article distributed under the terms of the Creative Commons Attribution License, which permits unrestricted use, distribution, and reproduction in any medium, provided the original author and source are credited. 